\title{
Alacranes (Arachnida: Scorpiones) de Frontera Corozal, en la selva lacandona, Chiapas, México, con la descripción de una nueva especie de Diplocentrus (Diplocentridae)
}

\section{Scorpions (Arachnida, Scorpiones) from Frontera Corozal, in the Lacandona rainforest, Chiapas, México, with the description of a new species of Diplocentrus (Diplocentridae)}

Oscar F. Francke B.

Colección Nacional de Arácnidos, Departamento de Zoología, Instituto de Biología, Universidad Nacional Autónoma de México, Tercer circuito exterior s/n, Apartado postal 70-153, Coyoacán, 04510. México, D. F.

Correspondencia: offb@ibiologia.unam.mx

Resumen. Se realizaron recolectas diurnas y nocturnas en la Comunidad de Frontera Corozal, Municipio de Ocosingo, Chiapas, México. Se registran 3 especies de escorpiones, 2 de la familia Buthidae y una de la familia Diplocentridae. Centruroides schmidti Sissom es un nuevo registro para el estado de Chiapas y Diplocentrus chol es una especie nueva.

Palabras clave: Scorpiones, Buthidae, Diplocentridae, Centruroides, Diplocentrus chol, especie nueva, Ocosingo.

Abstract. Diurnal and nocturnal collecting in the community of Frontera Corozal, Municipio Ocosingo, Chiapas, Mexico, revealed three species of scorpions, two belonging to the family Buthidae and one to Diplocentridae. Centruroides schmidti Sissom is a new record for the state of Chiapas, and Diplocentrus chol is described as a new species.

Key words: Scorpiones, Buthidae, Diplocentridae, Centruroides, Diplocentrus chol, new species, Ocosingo.

\section{Introducción}

La escorpiofauna del estado de Chiapas, México es muy poco conocida y aún menos la de la selva lacandona. Lourenço y Sissom (2000) listan 7 especies para Chiapas; Beutelspacher (2000) registra 9 especies para Chiapas, mientras que para Baja California y Baja California Sur (con extensión territorial muy similar a Chiapas, de aproximadamente $72000 \mathrm{~km}^{2}$ ) registra 38 y 42 especies respectivamente. En la selva tropical mediana y alta de la lacandona en Chiapas, sólo se ha registrado una especie; mientras que para los estados de Quintana Roo y Yucatán se han registrado 5, y para Campeche 4 (Beutelspacher, 2000).

Los investigadores y alumnos de las Colecciones Nacionales de Ácaros y Arácnidos del Instituto de Biología de la Universidad Nacional Autónoma de México, desde 2004 hasta 2006, inclusive, han concentrado sus investigaciones taxonómicas en las reservas ecológicas de la comunidad Chol, Municipio de Ocosingo, Chiapas,

Recibido: 05 abril 2006; aceptado: 07 agosto 2006 cuyo asentamiento principal es la población de Frontera Corozal, ubicada a orillas del río Usumacinta, en la frontera con Guatemala y cerca de las famosas ruinas arqueológicas de Bonampak y Yaxchilán.

\section{Materiales y método}

Se utilizaron 3 técnicas básicas para el muestreo de arácnidos y en particular de los alacranes: 1) colecta manual diurna bajo piedras y troncos, 2) colecta nocturna utilizando lámparas portátiles de luz ultravioleta (U.V.) y 3) 25 trampas pit-fall colocadas en 5 localidades diferentes ( 5 por localidad) y recolectadas mensualmente de mayo a octubre de 2005.

El material para estudios morfológicos se preservó en alcohol etílico al $80 \%$ (las muestras para análisis moleculares se colocaron en alcohol al $96 \%$ y se enviaron al Museo Americano de Historia Natural de Nueva York, para futuros estudios filogenéticos). Los ejemplares se estudiaron, dibujaron y midieron con un microscopio estereoscópico Zeiss Stemi V 11, equipado con retícula micrométrica en oculares de $10 \mathrm{X}$ y con una cámara lúcida. 
Las fotografias con luz ultravioleta se tomaron utilizando el mismo microscopio y una cámara digital Nikon Coolpix 4600; se apagaron todas las luces del laboratorio y se usaron 2 lámparas portátiles marca Sanelec, cada una equipada con 2 tubos fluorescentes U.V. Las mediciones y terminología se basan en uso previo (Francke, 1977, Francke y Ponce-Saavedra, 2005).

Para la estimación del número de mudas necesarias para alcanzar la madurez sexual se utilizó el método indirecto (Francke y Sissom, 1984) con una progresión de Dyar de 1.3.

Los ejemplares que se utilizaron se encuentran depositados en la siguientes colecciones: Museo Regional de la Cuenca del Río Usumacinta (MRCRU), Frontera Corozal, Chiapas; American Museum of Natural History (AMNH), Nueva York, EUA; Colección Nacional de Arácnidos (CNAN), Instituto de Biología de la UNAM, D. F., México.

\section{Resultados}

La diversidad de arácnidos ha sido sorprendente; habiéndose colectado hasta el momento 10 de los 11 órdenes existentes (exceptuando Solifugae), 70 familias y 181 géneros, y el trabajo de campo y de laboratorio aún no ha concluido. Muy significativos han sido los hallazgos de 3 nuevas especies de palpígrados (Montaño, 2006; Montaño y Francke, 2006), una nueva especie de uropígido (Ballesteros y Francke, 2006), y 2 nuevas especies de esquizómidos (no publicados).

Se encontraron 3 especies de alacranes. Ninguna se considera de importancia médica o que represente peligro para la salud pública; 2 pertenecen a la familia Buthidae y una a la familia Diplocentridae; una de las Buthidae es registro nuevo para el estado de Chiapas y el diplocéntrido, una especie nueva para la ciencia.

\section{Familia Buthidae C. L. Kock, 1837}

Escorpiones con esternón triangular; dedo móvil de los quelíceros con 2 dientes ventrales; fémur de los pedipalpos con 11 tricobotrias, patela con 13 tricobotrias, ninguna de ellas en la cara ventral. Aproximadamente 73 géneros, 530 especies y 165 subespecies de distribución mundial (Fet et al., 2000).

\section{Género Centruroides Marx, 1890}

Es el único género de esta familia indudablemente presente en México. El género Darchenia Vachon, 1977, descrito con base en un ejemplar supuestamente de Yucatán, al parecer es de origen africano y está mal etiquetado (Lourenço, 1995). El género Tityopsis Armas, 1974, contiene 2 especies válidas de Cuba (Fet et al., 2000) y una especie supuestamente de Tehuantepec, Oaxaca, México, basada en un solo ejemplar (Armas y Martín-Frías, 1998) (se han hecho esfuerzos para localizar otros ejemplares en la región de Tehuantepec que han resultado infructuosos, E. Martín-Frías, com.pers.; por otra parte, el holotipo está en mal estado de conservación y su origen es cuestionable, $\mathrm{O}$. F. Francke B. obs. pers.). Este género incluye las especies de importancia médica en el país. Tiene aproximadamente 46 especies y 22 subespecies distribuidas desde el sur de los Estados Unidos de América (EUA) hasta el norte de Sudamérica (Fet et al., 2000); de las cuales 22 especies y 9 subespecies están presentes en México.

\section{Centruroides gracilis (Latrielle, 1804)}

Diagnosis y comentarios. Escorpión negro o café muy oscuro, grande (adultos $10 \mathrm{~cm}$ o más), con 9 hileras de gránulos en el margen interno de los dedos de los pedipalpos. Especie de amplia distribución, desde EUA (Florida), todo Centroamérica, las Antillas Mayores, Colombia y Venezuela. En nuestro país ocupa las llanuras costeras del Golfo de México desde Tamaulipas hasta la península de Yucatán (Fet et al., 2000). Previamente se había registrado para Chiapas y para la selva lacandona (Buenos Aires, ruinas de Bonampak) por Beutelspacher (2000). Teruel y Stockwell (2002) publican fotografías a color de esta especie.

Nuevos registros: $2 \mathrm{~km}$ SE Frontera Corozal (150m), 28-IV-04 [16 48’12.7" N-90 52’11.2" O] 3machos, 1 hembra (CNAN) (luz U.V.) (R. Paredes y J. L. Castelo cols.); $4.1 \mathrm{~km}$ hacia El Tornillo (130m), 2-V-04 [16 ${ }^{0}$ 47'11.4" N-90 51'19.8" O] 1 juv. (CNAN) (diurna) (J. L. Castelo col.); $4.5 \mathrm{~km}$ N Crucero a Frontera Corozal (171m), 20-VIII-04 [16 $46^{0} \quad 47.3^{\prime \prime} \quad \mathrm{N}-90^{\circ} \quad 58^{\prime} 01.5^{\prime \prime}$ O] 1 hembra, 1 juv. $2^{\circ}$ estadio (CNAN) (luz U.V.) (R. Paredes col.); km 133 Frontera-Palenque (187m), 12-X04 [16 47'30.6" N-910 02`27.7" O] 3 machos (CNAN) (luz U.V.) (R. Paredes, J. L. Castelo y A. Ibarra cols.); El Taller, Sierra de La Cojolita $(257 \mathrm{~m}), 7-\mathrm{IV}-05$ [16 ${ }^{0}$ 45.756' $\left.\mathrm{N}-91^{0} 01.933^{\prime} \mathrm{O}\right] 5$ hembras (CNAN) (diurna) (O. Francke, A. Valdez y J. Ballesteros cols.); La Galleta (169m), 7-IV-05 [16 47.175' N-90 56.008' O] 1 macho +1 hembra (MRCRU), 2 hembras, 1 juv. (CNAN) (luz U.V.) (O. Francke, A. Valdez y J. Ballesteros cols.); Reserva de Lacandonia schismatica (167m), 6-VIII-05 [16 $\left.45.572^{\prime} \mathrm{N}-91^{0} 0.441^{\prime} \mathrm{O}\right] 1$ macho, 2 hembras, 1 juv $2^{\circ}$ estadio (CNAN) (luz U.V.) (A. Valdez y J. L. Castelo cols.); Arroyo Nayte, Sierra de La Cojolita (214m), 7-VIII05 [16 47.589 $\left.{ }^{\prime} \mathrm{N}-91^{0} 02.603^{\prime} \mathrm{O}\right] 1$ hembra (CNAN) (luz U.V.) (A. Valdez y J. L. Castelo cols.); Teca, $5 \mathrm{~km} \mathrm{O}$ Frontera Corozal (221m), 8-VIII-05 [16 $47.189^{\prime} \mathrm{N}-90^{0}$ 55.034' O] 1 hembra (CNAN) (diurna) (A. Valdez, J. L. Castelo y R. Paredes cols.); El Taller, Sierra de La Cojolita 
(186m), 9-VIII-05 [16 $\left.16^{0} 45.970^{\prime} \mathrm{N}--91^{0} 01.705^{\prime} \mathrm{O}\right] 1$ juv. $2^{\circ}$ estadio (CNAN) (diurna) (A. Valdez, A. Ávila y G. Montiel cols.); Reserva de La Lacandona (157m), 11VIII-05 [16 $\left.45.507^{\prime} \mathrm{N}-91^{0} 0.332^{\prime} \mathrm{O}\right] 1$ macho, 1 hembra (AMNH) (diurna) (A. Valdez, R. Paredes, A. Avila y G. Montiel cols.); El Encaño, Sierra de La Cojolita (165m), 5-IX-05 [ [ $16^{0} 48.712^{\prime} \mathrm{N}-91^{0}$ 04.460' O] 1 macho, 1 hembra (CNAN) (diurna) (O. Francke, M. Córdiva, A. Jaimes, A. Valdez y H. Montaño cols.); El Aserradero, 17 km O Frontera Corozal (207m), 6-IX-05 [16 47.080' $\left.\mathrm{N}-91^{\circ} 02.243^{\prime} \mathrm{O}\right] 1$ macho, 1 hembra con dos juv. $2^{\circ}$ estadio sobre el dorso (CNAN) (diurna) (O. Francke, M. Córdova, A. Jaimes, A. Valdez y H. Montaño cols.); El Cartón (153m), 6-IX-05 [16 $47.185^{\prime} \mathrm{N}-90^{0} 55.684^{\prime} \mathrm{O}$ ] 1 hembra (CNAN) (diurna) (O. Francke, M. Córdova, A. Jaimes, A. Valdez y H. Montaño cols.); El Taller, Sierra de La Cojolita (257m), 7-IX-05 [16 $1^{0} 45.756^{\prime}$ N-- 91 ${ }^{0}$ 01.933' O] 2 machos juvs. (CNAN)(diurna) (O. Francke, M. Córdova, A. Jaimes, A. Valdez y H. Montaño cols.); El Cartón (130m), 2-X-05 [16 47.471' N-90 55.719' O] 4 hembras (CNAN) (luz U.V.) (I. Mondragón, H. Montaño y G. Montiel cols.); Reserva Comunal La Cruz (218m), 4$\mathrm{X}-05\left[16^{0} 43.718^{\prime} \mathrm{N}-90^{\circ} 55.852^{\prime} \mathrm{O}\right] 2$ hembras (CNAN) (luz U.V.) (I. Mondragón, H. Montaño y G. Montiel cols.).

Variación. La variación encontrada en el número de dientes pectíneos en machos $(\mathrm{n}=13)$ fue: 30 dientes (3 peines), 31 (12), 32 (5), 33 (6); y en hembras $(n=24)$ fue: 28 dientes (7 peines), 29 (17), 30 (17) y 31 (6).

Historia natural. Esta especie se encontró con frecuencia, pero en bajas densidades. Habita en el suelo o cerca. Durante el día se recolectaron tanto adultos como juveniles, generalmente bajo piedras o troncos caídos. Con lámparas U.V. durante la noche se encontraron adultos, usualmente en el suelo, acechando presas cerca de sus madrigueras (en zonas rocosas donde se refugian en grietas y otras oquedades). Ningún ejemplar cayó en las trampas pit-fall. Ciclo de vida. Esta especie fue criada en cautiverio en un estudio previo (Francke y Jones, 1982). Las hembras alcanzan la madurez sexual en el $7^{\circ}$ estadio. Los machos tienen crecimiento alométrico del segmento caudal $\mathrm{V}$ en la última muda, y pueden alcanzar la madurez sexual en el $6^{\circ}$ o $7^{\circ}$ estadio, es decir, que existen machos adultos "pequeños" y "grandes" en una misma camada. Durante este estudio se colectaron varios ejemplares de $2^{\circ}$ estadio en agosto y septiembre, lo cual podría indicar que el parto en esta especie está ligado al inicio de la temporada de lluvias en esta región del país.

\section{Centruroides schmidti Sissom, 1995}

Diagnosis y comentarios. Alacrán pequeño y esbelto (adultos 3-4 cm); de color paja, muy manchado de negro (pero no rayado); con 8 filas de gránulos en el borde interno de los dedos de la quela de los pedipalpos. En Teruel y Stockwell (2002) pueden verse fotografías a color de esta especie.

Es una especie sumamente rara, descrita hace poco más de una década con base en 2 ejemplares: un macho adulto de Honduras y una hembra de Guatemala (Sissom, 1995). Posteriormente, se registraron un macho y 3 hembras para Quintana Roo (Armas, 1996) y 22 ejemplares de 3 localidades diferentes en Honduras (Teruel y Stockwell, 2002).

Nuevos registros: El Tornillo, $2 \mathrm{~km}$ SE Frontera Corozal (150m), 28-IV-04 [16 48'12.7" N-90 52'11.2" O] 1 hembra (CNAN) (luz U.V.) (R. Paredes y J. L. Castelo cols.); Puente de Agua Azul (110m), 7-IV-05 [16 48.308' $\left.\mathrm{N}-90^{\circ} 54.417^{\prime} \mathrm{O}\right] 2$ machos, 1 hembra (CNAN) (luz U.V) (O. Francke, A. Valdez y J. Ballesteros cols.); La Galleta (164m), 7-IV-05 [16 47.175' N-90 56.008' O] 1 macho (CNAN) (luz U.V.) (O. Francke, A. Valdez y J. Ballesteros cols.); Puente de Agua Azul (110m), 8-IV05 [ $\left.16^{0} 48.260^{\prime} \mathrm{N}-90^{\circ} 54.053^{\prime} \mathrm{O}\right] 1$ macho (MRCRU), 1 macho, 1 hembra (AMNH), 4 machos, 2 hembras (una cargando una cría de $2^{\circ}$ estadio sobre el dorso) (CNAN) (luz U.V.) (O. Francke, A. Valdez y J. Ballesteros cols.). Variación. La variación encontrada en el número de dientes pectíneos en machos ( $\mathrm{n}=9$ ) fue: 13 dientes ( 2 peines), 14 (10), 15 (4) y 16 (1) (1 peine dañado); y en hembras $(n=5)$ fue: 12 dientes (6 peines), 13 (3) y 14 (1). Las poblaciones de Honduras tienden a un conteo mayor, en machos la moda es 15 (rango 12 a 15) y en hembras 14 (rango 13 a 15) (Teruel y Stockwell, 2002).

Historia natural. La aparente "rareza" de esta especie puede deberse a su hábitat y a su comportamiento antidepredadores. Se encontró exclusivamente durante las recolectas nocturnas con lámparas ultravioleta, generalmente en ramitas de $1 \mathrm{a} 2 \mathrm{~cm}$ de diámetro, entre 1 y $4 \mathrm{~m}$ de altura (normalmente se ilumina el suelo para ver donde se camina y para localizar alacranes de hábitos terrestres). Son animales sumamente sensibles y a la menor perturbación (como el tocar la rama para tratar de alcanzarlos) se dejan caer al suelo donde rápidamente desaparecen entre la hojarasca (cerca del $30 \%$ de los ejemplares localizados escaparon de esta manera y otro tanto permaneció en ramas altas fuera de nuestro alcance). Los ejemplares de Honduras también fueron recolectados de noche con lámparas U.V., principalmente en ramas de arbustos.

Ciclo de vida. Por la rareza de esta especie se desconocen detalles de su ciclo de vida. En la estimación del número de mudas se observa que en las hembras el crecimiento del segmento caudal $\mathrm{V}$ es isométrico con la longitud del carapacho y que probablemente maduran en el $6^{\circ}$ estadio 
(Fig. 1). En los machos el crecimiento del segmento V es marcadamente alométrico y la madurez sexual se alcanza probablemente en el $5^{\circ}$ o $6^{\circ}$ estadio; es decir que al igual que en la especie anterior, posiblemente existan machos adultos con dos tallas corporales diferentes.

Familia Diplocentridae Karsch, 1880

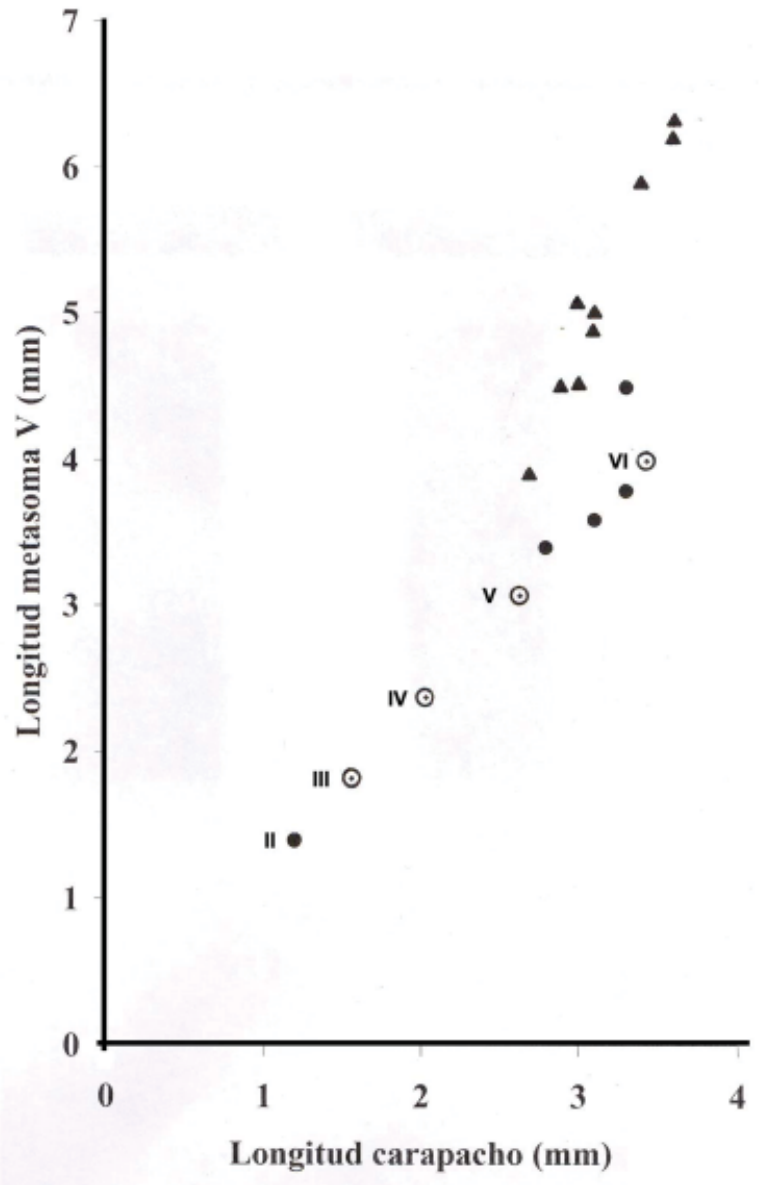

Figura 1. Morfometría de Centruroides schmidti Sissom: círculos sólidos $=$ hembras, triángulos $=$ machos, círculos abiertos con punto=estadios predichos (III, IV, V y VI) en el ciclo de vida a partir del estadio II (observado) hasta la madurez sexual.

Escorpiones con esternón pentagonal; dedo móvil de los quelíceros sin dientes ventrales; fémur de los pedipalpos con 3 tricobotrias, patela con 19 tricobotrias, 3 de ellas en la cara ventral. Incluye 7 géneros y unas 80 especies; tiene una distribución geográfica discontinua 2 géneros y unas 8 especies en el Cercano Oriente y 6 géneros y las demás especies en el Nuevo Mundo (un género compartido en ambas áreas) (Fet et al., 2000).

En México existen unas 40 especies descritas para esta familia (en 2 géneros) y se conocen otras 20 aún por describir. La mayoría de las especies corresponden al género Diplocentrus Peters. Hay 4 especies de Baja California Sur (y 1 descrita recientemente de Guerrero) que han sido asignadas al género Bioculus Stahnke, 1968, cuya validez ha sido previamente cuestionada (Francke, 1978; Lourenço y Sissom, 2000; Fet et al., 2000) por carecer de caracteres diagnósticos propios (=autopomorfia genérica).

\section{Género Diplocentrus Peters, 1861}

Alacranes de talla variable (adultos 2.5 a $15 \mathrm{~cm}$ ), con las quelas de los pedipalpos muy desarrolladas y robustas; los telotarsos de las patas armados ventralmente con 2 filas de sedas espiniformes; telson con un prominente tubérculo redondeado y sedoso bajo el aguijón; borde interno de los dedos de los pedipalpos con 6 subfilas de gránulos. El género de distribuye desde el sur de EUA hasta Honduras; ampliamente distribuido en la República Mexicana (no existen registros para los estados de Baja California, Colima, Tlaxcala y el D. F.).

Diplocentrus chol nueva especie (Figuras 2-13)

Diagnosis. Tamaño mediano, adultos 45-50 mm de largo total. Quelíceros con dedo fijo más corto que el ancho de la quela, dedo móvil más corto que el largo de la quela. Ortobotriotaxia tipo C . Fémur de los pedipalpos más ancho que alto, cara dorsal plana. Dientes pectíneos: machos 1013 (moda 11), hembras 8-12 (moda 10). Formula global de espinas telotarsales 4/4-5; 4-5/5; 5/6; 5-6/6. Cápsula del hemiespermatóforo con tres lóbulos, el lóbulo interno claramente sigmoideo.

El conteo pectinal junto con la fórmula telotarsal sirven para distinguir a esta especie de cualquier otra en el género (véanse comentarios taxonómicos más adelante).

Descripción del holotipo. Color dorsal café a caférojizo, vientre y patas amarillentas; carapacho y tergitos densamente jaspeados con fuscosidad café oscuro, patas marmoleadas.

Prosoma. Carapacho con 3 pares de ocelos laterales; ojos medios en el $30 \%$ anterior; margen anterior con 5 pares de sedas, profundamente bilobado, muesca media en forma de V obtusa; triángulo interocular con granulación mediana, densa; áreas laterales coriáceas a densa y diminutamente granulosas. Esternón pentagonal, igual de ancho que de largo; con 10 pares de sedas simétricamente dispuestas. Mesosoma. Tergitos coriáceos a granulados densa y finamente, sin carenas; VII con gránulos gruesos en la mitad posterior, carenas submedianas y laterales poco definidas por gránulos mayores algo dispersos. Esternitos brillantes, lisos a finamente punteados; estigmas respiratorios oblicuos, alargados; VII con carenas submedianas obsoletas, carenas laterales débiles a vestigiales, lisas. Opérculos genitales con 3 sedas cada uno; papilas genitales prominentes. 
Metasoma. Segmentos I a IV con carenas dorsolaterales bien desarrolladas, granulosas; carenas laterales supramedianas fuertes, granulosas; carenas laterales inframedianas moderadas y granulosas, excepto en el IV débiles, con gránulos dispersos; carenas ventrolaterales moderadas a fuertes, en I crenuladas, en II casi lisas, en III y IV serrilladas; carenas ventrales submedianas en I a III muy débiles a vestigiales, en I y II lisas, en III con pocos granulitos, en IV débiles a medianas, con gránulos medianos más densos. Segmento V con carenas dorsolaterales fuertes, granulosas; carenas lateromedianas débiles basalmente y desapareciendo en el tercio medio, con granulación dispersa; carenas ventrolaterales, ventromediana y ventrotransversal fuertes, con grandes gránulos cónicos. Telson con vesícula densamente pilosa. Quelíceros. Base amarillo pálido, oscureciendo distalmente; muy jaspeados dorsalmente. Dedo móvil con dientes distales subiguales, oponibles (Fig. 2).

Pedipalpos. Fémur (Fig. 3) más ancho que alto, cara dorsal casi plana y moderadamente granulosa; carenas dorsointerna, dorsoexterna y ventrointerna fuertes, granulosas. Patela (Figs. 4-5) con carenas dorsointerna y ventrointerna pobremente definidas basalmente por algunos gránulos dispersos; carenas dorsomediana, dorsoexterna y ventroexterna muy fuertes, lisas; cara interna con granulación mediana, densa; caras dorsal y externa con reticulación media bien definida. Quelas (Figs. 6-7) muy robustas, casi del doble de alto que de ancho; carena digital muy fuerte, lisa; carena dorsal marginal fuerte, escabrosa (gránulos romos superimpuestos en la reticulación); carena dorsal secundaria moderada a fuerte, lisa; carena secundaria externa débil a vestigial, lisa; carena ventromediana muy fuerte, lisa, dirigida hacía el medio de la articulación del dedo móvil; carena ventrointerna fuerte, lisa; caras dorsal y externa con reticulación mediana muy marcada, las crestas lisas y brillantes, las celdas coriáceas.

Patas. Fórmula de espinas del telotarso: 4/5 5/5; 5/5 5/5; 5/6 5/6; 6/6 5/6.

Hemiespermatóforo (Figs. 8-11). Lámina angosta y larga. Zona capsular con 3 lóbulos: lóbulo externo con una fuerte cresta, muy esclerosada, irregularmente crenulada; lóbulo medio poco esclerosado, inconspicuo entre los lóbulos externo e interno (Fig. 10); lóbulo interno sigmoideo en vista ental (Fig. 11).

Medidas del holotipo (en mm; L=largo, $\mathrm{A}=$ ancho, $\mathrm{a}=$ alto [dorso-ventral]). L total 45.5. L Carapacho 5.7, A máximo 5.4. L Mesosoma 13.6. Metasoma: I L/A 3.3/3.3; II L/A 3.5/3.0; III L/A 3.8/3.0; IV L/A 4.5/2.8; V L/A 6.0/2.5; Telson L/A/a 5.1/2.5/2.0. Quelícero L/A 1.9/1.2, $\mathrm{L}$ dedo fijo 0.8, L dedo móvil 1.5. Pedipalpo: fémur L/ A/a 5.2/2.0/1.4; patela L/A/a 5.5/2.2/2.2; quela L/A/a 10.6/2.7/5.2, L ventral 5.0; L dedo fijo 4.5; L dedo móvil 6.1. Dientes pectíneos 12-11 (derecho-izq.).

Variación. El género Diplocentrus se caracteriza por el dimorfismo sexual en la quela de los pedipalpos de los ejemplares adultos; los machos generalmente presentan quelas angulares, con carenas bien desarrolladas y con escultura cuticular en las caras dorsal y externa, mientras

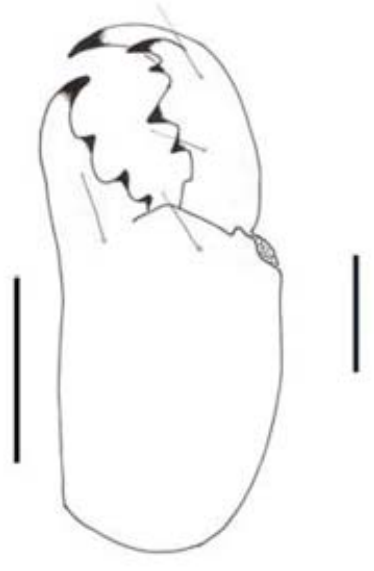

2

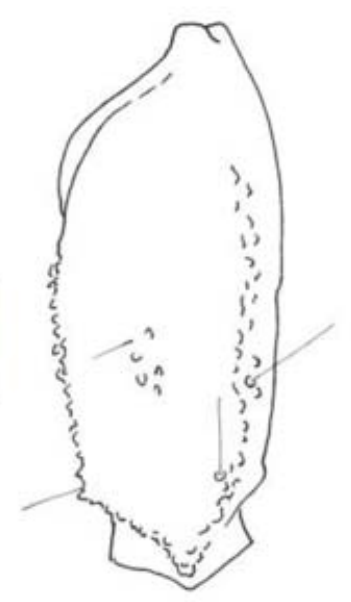

3

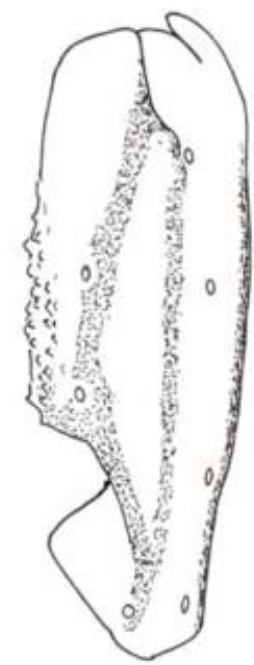

4

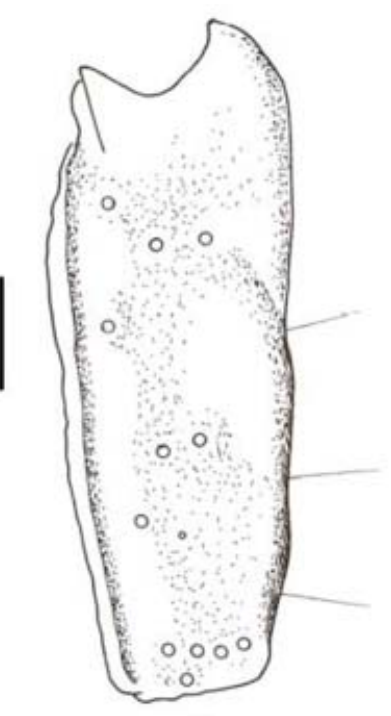

5

Figuras 2-5. Diplocentrus chol, nueva especie de la selva lacandona, Chiapas, México. 2, quelícero derecho, dorsal; 3, fémur del pedipalpo, dorsal; 4, patela del pedipalpo, dorsal; 5 , patela del pedipalpo, externa (escalas=1 mm). 


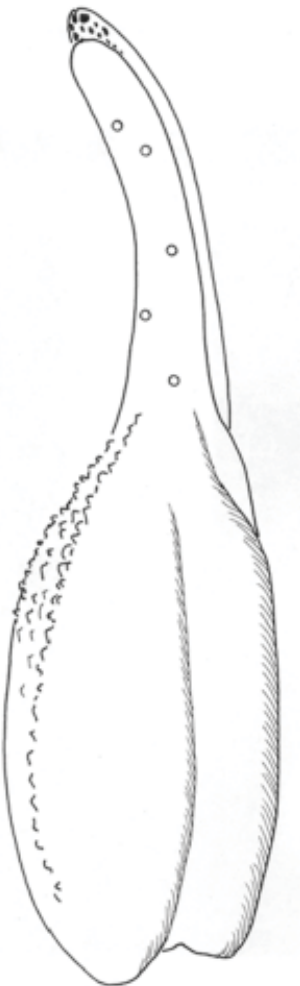

6
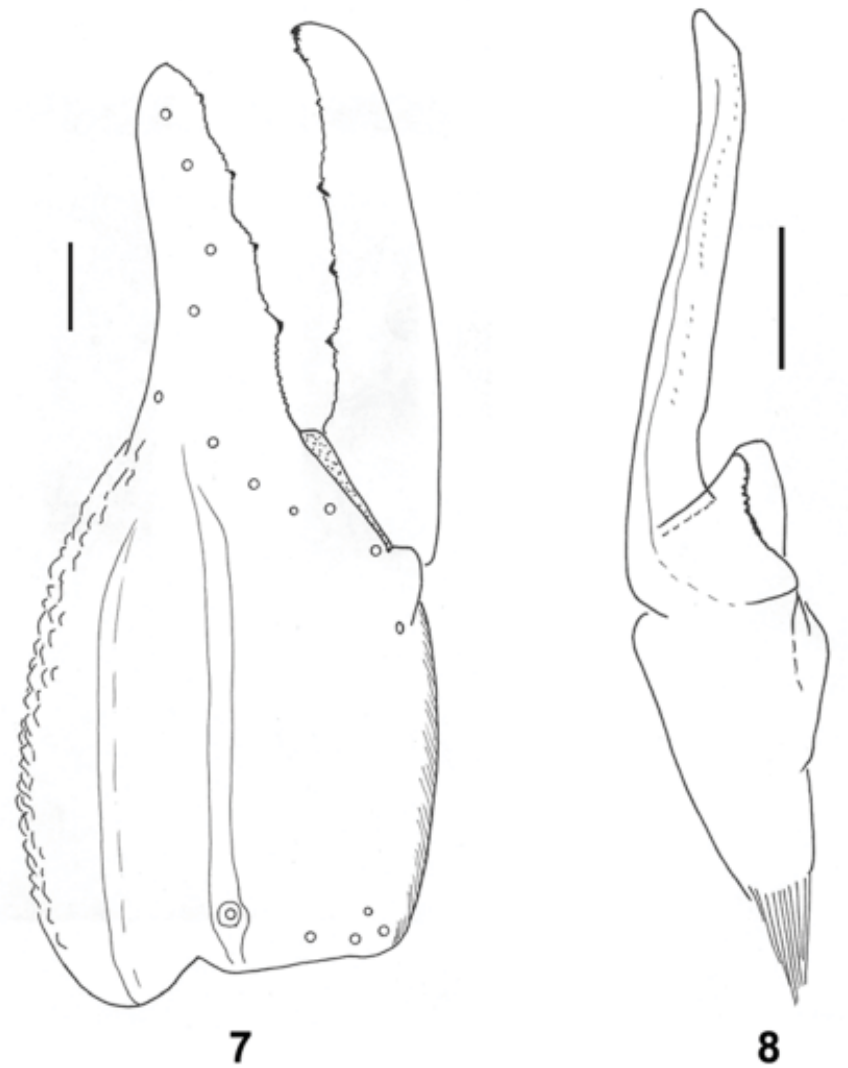

Figuras 6-8. Diplocentrus chol, nueva especie de la selva lacandona, Chiapas, México. 6, quela del pedipalpo, dorsal; 7, quela del pedipalpo, externa; 8 , hemiespermatóforo, dorsal (escalas=1 mm).

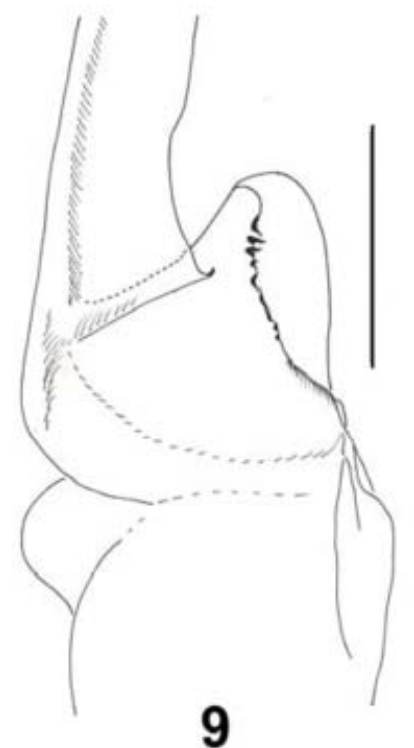

9

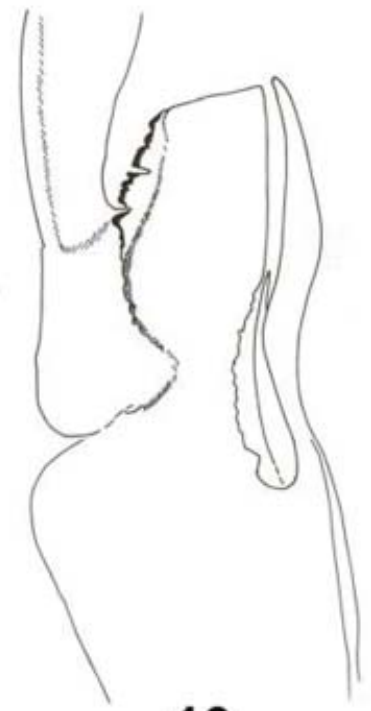

10

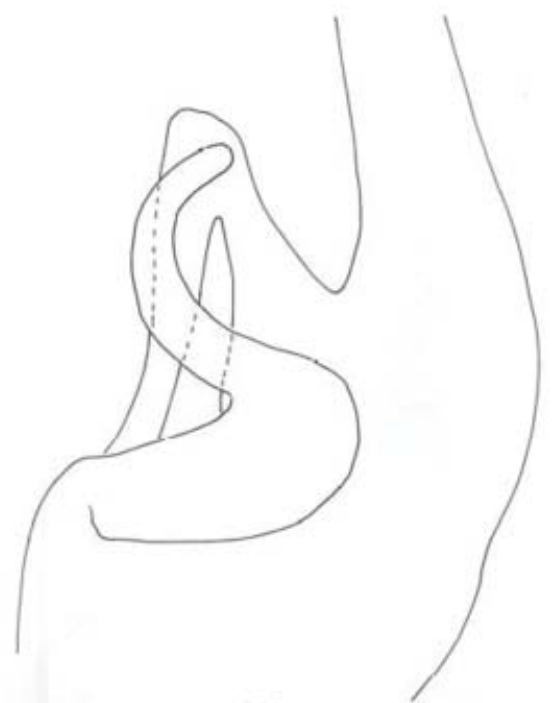

11

Figuras 9-11. Diplocentrus chol, nueva especie de la selva lacandona, Chiapas, México. Detalles de la región capsular del hemiespermatóforo. 9, dorsal; 10, dorso-ental; 11, ental (escala=1 mm). 


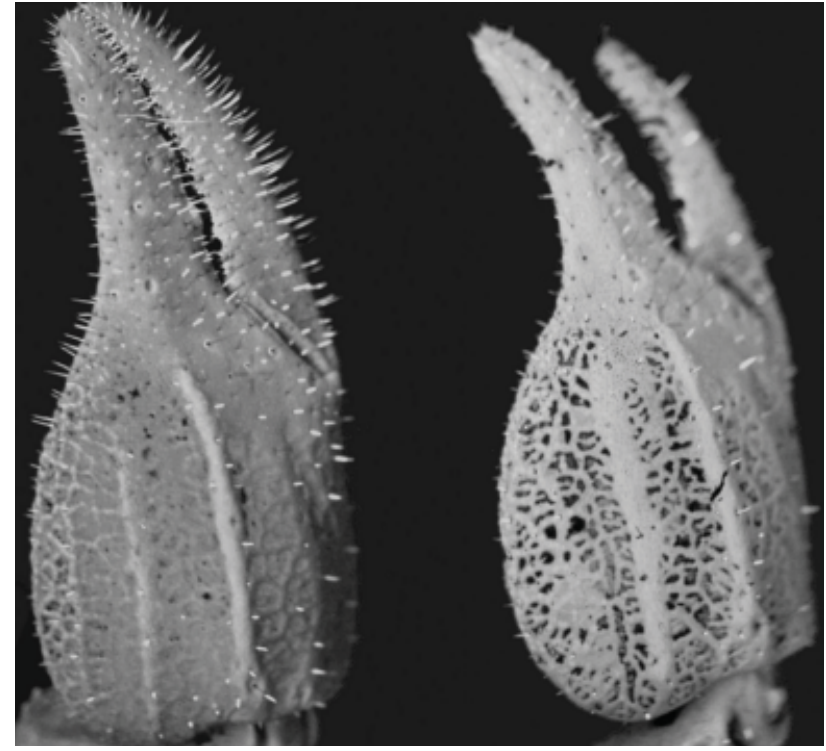

Figuras 12-13. Diplocentrus chol, nueva especie de la selva lacandona, Chiapas, México. Quela de los pedipalpos mostrando dimorfismo sexual marcado en densidad de sedas, leve en forma y casi ausente en escultura cuticular. 12, macho adulto (izquierda); 13, hembra adulta (derecha). Fotos tomadas con luz ultravioleta.

que en las hembras las quelas son redondeadas, con carenas débiles o vestigiales y las caras dorsal y externa lisas (Francke, 1977). Es inusual el dimorfismo sexual mínimo de Diplocentrus chol; la quela de la hembra es poco más inflada y globosa que en el macho, pero el desarrollo de las carenas y la escultura cuticular son casi iguales (Figs. 12-13).

La variación en dientes pectíneos en machos $(n=11)$ fue: 10 dientes (1 peine), 11 (16; moda con 73\%), 12 (4) y $13(1)$; en hembras $(\mathrm{n}=26) 8$ dientes (1 peine), $9(1), 10$ (30; moda con $59 \%), 11(17 ; 33 \%)$ y $12(2)$.

Espinas telotarsales $(n=70)$. Fila prolateral: pata I con 4 espinas (97\%), II con 4 (36\%) o $5(64 \%)$ espinas, III con $5(87 \%)$ espinas, y IV con $5(50 \%)$ o $6(50 \%)$ espinas. Fila retrolateral: pata I con $4(34 \%)$ o $5(66 \%)$ espinas, II con 5 espinas (98\%), III con 5 espinas (87\%) y IV con 6 espinas (99\%). La fórmula global para la especie dada en la diagnosis refleja la variabilidad encontrada en las filas prolaterales II y IV, y retrolateral I.

\section{Resumen taxonómico}

Serie típica. Holotipo macho (CNAN T-0214) y 3 paratipos (macho subadulto, macho juvenil, hembra juvenil) (CNAN T-0215) de Chiapas, Municipio de Ocosingo, Comunidad de Frontera Corozal, Arroyo Nayte, Reserva Comunal Sierra de la Cojolita (206m), 8-IX-2005 [16 ${ }^{0}$ 47.642'
$\left.\mathrm{N}-91^{0} 02.538^{\prime} \mathrm{O}\right]$ (diurna) (O. Francke, M. Córdova, A. Jaimes, A. Valdez y H. Montaño cols.); misma localidad, 6 hembras y 1 macho juv. paratipos (CNAN T-0217), 9-IV05 (diurna) (O. Francke, A. Valdez y J. Ballesteros cols.); misma localidad, 1 hembra paratipo (CNAN T-0229), 4-VI-05 (diurna) (O. Francke, G. Villegas, G. Montiel y S. Rubio cols.); misma localidad, 1 hembra subadulta, 2 hembras juvs. paratipos (CNAN T-0218), 8-VIII-05 (diurna) (J. L. Castelo, R. Paredes y G. Montiel cols.); misma localidad, 1 macho juv. paratipo (CNAN T-0224), 9-VIII-05 (diurna) (A. Gudiño col.); misma localidad, 8IX-05, 1 macho juv. paratipo (CNAN T-0225) (diurna) (O. Francke, M. Córdova, A. Jaimes, A. Valdez y H. Montaño cols.); misma localidad, 2 machos, 3 hembras, 1 hembra subadulta, 2 juvs. paratipos (CNAN T-0216), 3-X-05 (diurna) (H. Montaño, G. Montiel y I. Mondragón cols.); El Encaño (165 m), 8-IV-05 [16 $48.677^{\prime} \mathrm{N}-91^{0} 04.646^{\prime}$ O], 1 macho, 1 hembra, 1 macho subadulto, 1 hembra juv. paratipos (CNAN T-0220) (diurna) (O. Francke, A. Valdez y J. Ballesteros cols.); misma localidad, 7-IX05, 1 hembra, 1 macho juv. paratipos (CNAN T-0219) (diurna) (O. Francke, M. Córdova, A. Jaimes, A. Valdez y H. Montaño cols.); misma localidad, 3-X-05, 1 hembra paratipo (CNAN T-0227) (diurna) (H. Montaño, G. Montiel y I. Mondragón cols.); El Taller, Reserva Comunal Sierra de la Cojolita (183 m), VIII-04 [16 46'01.1" N-910 01' 42.6" O], 1 hembra paratipo (AMNH T-0230) (diurna) (G. Montiel col.); El Taller, Reserva Comunal Sierra de la Cojolita (257 m), 7-IV-05 [16 $6^{0} 45.756^{\prime} \mathrm{N}-91^{0} 01.933^{\prime}$ O], 1 macho juv. paratipo (CNAN T-0231)(diurna) (O. Francke, A. Valdez y J. Ballesteros cols.); El Taller (186 m), 9-VIII-05 [16 $\left.16^{0} 45.470^{\prime} \mathrm{N}-\mathrm{N}^{\circ} 01^{0} 01.705^{\prime} \mathrm{O}\right], 1$ hembra juv. paratipo (CNAN T-0221)(diurna) (A. Valdez, G. Montiel y A. Ávila cols.); Reserva Comunal La Cruz (218 m), 4-

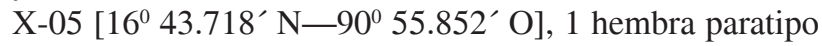
( cargando 2 crías de $2^{\circ}$ estadio en el dorso) (CNAN T0222) (diurna) ( H. Montaño y G. Montiel cols.); misma localidad y fecha, 1 hembra paratipo (CNAN T-0223) (luz U.V.) (H. Montaño y G. Montiel cols.); Reserva de Lacandonia schismatica (213 m), 11-IV-05 [16 44.798' $\left.\mathrm{N}-91^{0} 00.757^{\prime} \mathrm{O}\right], 1$ hembra paratipo (CNAN T-0228) (diurna) (O. Francke, A. Valdez y J. Ballesteros cols.).

Etimología. Esta especie está dedicada a la comunidad indígena Chol, habitantes de la región de la selva lacandona, comprendida dentro de este estudio.

\section{Comentarios taxonómicos}

Las especies geográficamente más cercanas a Diplocentrus chol y con las cuales se podría confundir a la nueva especie son: Diplocentrus chiapasensis 
Beutelspacher et Armas, 1998; Diplocentrus montecristo Armas et Martín-Frías, 2000; Diplocentrus sinaan Armas et Martín-Frías, 2000; y Diplocentrus steelae Stockwell, 1988; pero la nueva especie puede diferenciarse claramente de cada una de ellas por las siguientes características:

1. Diplocentrus chiapasensis se conoce de un solo ejemplar juvenil hembra de una cueva de las inmediaciones de Ocozocuautla, Chiapas. Dicha hembra posee 13-14 dientes pectíneos y la fórmula telotarsal de 4/4; 4/5; 4/5; 5/5: las hembras de $D$. chol tienen 10-11 (92\%) dientes y en las patas III 5/6 espinas telotarsales.

2. Diplocentrus montecristo sólo se conocen 2 machos de la localidad de Montecristo, Municipio de Villaflores, Chiapas. Dichos ejemplares tienen 10-11 y 12-11 dientes pectíneos (moda $=11$, igual que en la nueva especie), $\mathrm{y}$ una fórmula telotarsal de $4 / 4 ; 4 / 4 ; 5 / 5 ; 5 / 5$, y difiere de la nueva especie en las espinas retrotarsales en las patas II $(\mathrm{n}=5)$, III $(\mathrm{n}=6)$ y IV $(\mathrm{n}=6)$.

3. Diplocentrus sinaan del Cañón del Sumidero en Chiapas, es de mayor tamaño; el macho tiene 16-16 dientes pectíneos y la hembra 14-14; la fórmula telotarsal es de $5 / 5 ; 5 / 5 ; 6 / 6 ; 6 / 6$, y difiere de la nueva especie en el número de espinas prolaterales I $(n=4)$ y III $(n=5)$.

4. Finalmente, Diplocentrus steelae sólo se conoce un macho adulto de La Victoria, Chiapas (municipio y/o coordenadas geográficas desconocidas; hay un mínimo de 6 localidades con ese nombre en dicha entidad); de 25 $\mathrm{mm}$ de longitud (versus 45-50 $\mathrm{mm}$ en la nueva especie), coloración amarillenta a café-rojizo sin fuscosidad (con fuscosidad jaspeada y densa en la nueva especie); segmento metasomal I más largo que ancho (proporciones iguales en la nueva especie); el dedo fijo de la quela de los pedipalpos proporcionalmente más corto (proporción largo quela/largo dedo de 2.65 versus 2.33 en la nueva especie); y tiene el lóbulo interno de la cápsula del hemiespermatóforo ancho y recto (versus delgado y sigmoideo en la nueva especie).

\section{Agradecimientos}

Deseo agradecer a la comunidad y a la Asociación Civil de Frontera Corozal por permitirnos el acceso, y a los guías que nos asignaron, por su invaluable ayuda en el trabajo de campo. A los siguientes colaboradores de las Colecciones Nacionales de Ácaros y Arácnidos, por el compañerismo y esfuerzo: A. J. Ballesteros, J. L. Castelo, M. Córdova, C. Durán, A. Jaimes, I. Mondragón, H. Montaño, G. Montiel, R. Paredes, S. Rubio, A. Valdez y G. Villegas. Gracias también a M. Córdova y R. Paredes por su ayuda con las ilustraciones, y a G. Montiel-Parra, G. VillegasGuzmán, M. del C. Guzmán-Cornejo, Rolando Teruel y a dos evaluadores anónimos por sus comentarios sobre el manuscrito. Finalmente, este trabajo fue posible gracias al apoyo económico del CONACYT (proyecto No. COI043/B1 de la Dra. Elena Álvarez-Buylla), del Instituto de Ecología de la UNAM, y de la National Science Foundation de los Estados Unidos (proyecto NSF BIO-DEB 0413453 del Dr. Lorenzo Prendini).

\section{Literatura citada}

Armas, L. F. de. 1996. Presencia de Centruroides schmidti Sissom en el sureste de México y descripción de dos especies nuevas (Scorpiones: Buthidae). Revista Nicaragüense de Entomología 36:21-33.

Armas, L. F. de y E. Martín-Frías. 1998. Presencia del género Tityopsis en México y descripción de una especie nueva (Scorpiones: Buthidae). Anales de la Escuela Nacional de Ciencias Biológicas (México) 43:45-49.

Ballesteros, J. A. y O. F. Francke. 2006. Mastigiproctus lacandonensis, especie nueva de vinagrillo (Thelyphonida, Arachnida) de la selva Lacandona, Chiapas, México. Entomología Mexicana 5:156-161.

Beutelspacher, C. R. 2000. Catálogo de los alacranes de México. Universidad Michoacana de San Nicolás de Hidalgo, Morelia, Michoacán. 175 p.

Fet, V., W. D. Sissom, G. Lowe y M. E. Braunwalder. 2000. Catalog of the scorpions of the world (17581998). New York Entomological Society. 690 p.

Francke, O. F. 1977. Scorpions of the genus Diplocentrus from Oaxaca, Mexico (Scorpionida, Diplocentridae). Journal of Arachnology 4:145-200.

Francke, O. F. 1978. Systematic revision of diplocentrid scorpions from circum-Caribbean lands. Special Publications of Texas Tech University 14:1-92.

Francke, O. F. y S. K. Jones. 1982. The life history of Centruroides gracilis (Scorpiones, Buthidae). Journal of Arachnology 10:223-239.

Francke, O. F. y J. Ponce-Saavedra. 2005. A new species of Diplocentrus (Arachnida: Scorpiones) from Michoacán, Mexico. Revista Mexicana de Biodiversidad 76:49-53.

Francke, O. F. y W. D. Sissom. 1984. Comparative review of the methods used to determine the number of molts to maturity in scorpions (Arachnida), with analysis of the post-birth development of Vaejovis coahuilae Williams (Vaejovidae). Journal of Arachnology 12:1-20.

Lourenco, W. R. 1995. Darchenia, a genus of scorpion which was not American. Biogeographica 71(4):197-199.

Lourenco, W. R. y W. D. Sissom. 2000. Scorpiones. In Biodiversidad, taxonomía y biogeografía de artrópodos de México: hacia una síntesis de su conocimiento, 
vol. II, J. E. Llorente Bousquets, E. G. Soriano y N. Papayero (eds.) Universidad Nacional Autónoma de México, México, D.F.

Montaño, H. 2006. Contribución al conocimiento de la taxonomía y distribución de los palpígrados (Arachnida: Palpigradi) en México. Tesis, Facultad de Ciencias, Universidad Nacional Autónoma de México, México, D. F. 103 p.

Montaño, H. y O. F. Francke. 2006. Descripción de una nueva especie de palpígrado (Arachnida: Palpigradi) del género Eukoenenia, colectado en la selva Lacandona, en México. Entomología Mexicana 5:162-166.

Sissom, W. D. 1995. Redescription of the scorpion Centruroides thorelli Kraepelin (Buthidae) and description of two new species. Journal of Arachnology 23:91-99.

Teruel, R. y S. A. Stockwell. 2002. A revision of the scorpion fauna of Honduras, with the description of a new species (Scorpiones, Buthidae, Diplocentridae). Revista Ibérica de Aracnología 6:111-127. 